BARI-TH 350/99

\title{
DYNAMICAL SYMMETRY BREAKING IN PLANAR QED
}

\author{
Paolo Cea ${ }^{a, b}$ and Luigi Tedesco ${ }^{b}$ \\ ${ }^{a}$ Dipartimento di Fisica and ${ }^{b} \mathrm{INFN}$, Sezione di Bari \\ Via Amendola 173, I-70126 Bari, Italy*
}

\begin{abstract}
We investigate $(2+1)$-dimensional $Q E D$ coupled with Dirac fermions both at zero and finite temperature. We discuss in details two-components ( $\mathrm{P}$ odd) and four-components (P-even) fermion fields. We focus on P-odd and P-even Dirac fermions in presence of an external constant magnetic field. In the one-loop approximation we find that the spontaneous generation of an uniform magnetic condensate is energetically favoured in the case of the P-odd massive theory. Moreover, we find that the spontaneous generation of the magnetic condensate survives even at infinite temperature. We also discuss the spontaneous generation of fermion mass in presence of an external magnetic field.
\end{abstract}




\section{Introduction}

Three dimensional gauge theories have attracted interest since long time due to the possibility of a gauge invariant mass term for the gauge fields [1]. Three dimensional field theoretical models are believed to provide effective theories for long distance planar condensed matter models [2] and high temperature four dimensional gauge field theories [3]. In particular quantum electrodynamics in $(2+1)$-dimensions has been intensively studied within the effective theories of high- $T_{c}$ superconductivity [4].

As a matter of fact, it turns out that in $Q E D_{2+1}$ with massive Dirac fermions the perturbative ground state is unstable towards the spontaneous generation of an uniform magnetic condensate [5],6]. Moreover, it turns out that this remarkable property is not shared with other $(2+1)$-dimensional field theories [7].

It is well known that four dimensional Dirac fermions with Yukawa coupling to a scalar field develop zero mode solutions near a domain wall [8]. Indeed, let us consider a four dimensional massless Dirac fermion coupled to a scalar field through the Yukawa coupling :

$$
\mathcal{L}_{\psi \phi}=\mathcal{L}_{\psi}+\mathcal{L}_{\phi}
$$

where

$$
\begin{gathered}
\mathcal{L}_{\psi}=\bar{\psi}(x) i \not \partial \psi(x)-g_{Y} \bar{\psi}(x) \psi(x) \phi(x), \\
\mathcal{L}_{\phi}=\frac{1}{2}\left(\partial_{\mu} \phi\right)^{2}-\frac{\lambda}{4}\left(\phi^{2}-v^{2}\right)^{2} .
\end{gathered}
$$

The scalar field develops a non vanishing vacuum expectation value $\langle\phi\rangle= \pm v$. In general one may assume that there are regions with $\langle\phi\rangle=+v$ and $\langle\phi\rangle=-v$. It is easy to see that the classical equation of motion of the scalar field admits the solution describing the transition layer between two adjacent regions with different values of $\langle\phi\rangle$ :

$$
\phi(z)=v \tanh \left(\frac{z}{\Delta}\right)
$$

where 


$$
\Delta=\frac{1}{v \sqrt{\frac{\lambda}{2}}}
$$

is the thickness of the wall.

The solution Eq. (4) is known as kink. In presence of the kink the Dirac equation

$$
\left[i \not \partial-g_{Y} \phi(x)\right] \psi(x)=0
$$

admits zero energy solutions localized on the wall [8]. Using the following representation of the Dirac algebra:

$$
\gamma^{3}=i\left(\begin{array}{ll}
0 & 1 \\
1 & 0
\end{array}\right), \quad \gamma^{\alpha}=\left(\begin{array}{cc}
\tilde{\gamma}^{\alpha} & 0 \\
0 & -\tilde{\gamma}^{\alpha}
\end{array}\right), \quad \alpha=0,1,2
$$

with

$$
\tilde{\gamma}^{0}=\sigma_{3}, \quad \tilde{\gamma}^{1}=i \sigma_{1}, \quad \tilde{\gamma}^{2}=i \sigma_{2},
$$

we find for the zero modes:

$$
\begin{gathered}
\psi_{0}(x, y, z)=\frac{N}{\sqrt{2}} \omega(z)\left(\begin{array}{l}
\rho(x, y) \\
\rho(x, y)
\end{array}\right) \\
\omega(z)=\exp \left\{-g_{Y} \int_{0}^{z} \phi\left(z^{\prime}\right) d z^{\prime}\right\}
\end{gathered}
$$

where $\rho(x, y)$ is a Pauli spinor, and

$$
N=\left[\int_{-\infty}^{+\infty} d z \omega^{2}(z)\right]^{-\frac{1}{2}}=\sqrt{\pi} \Delta \frac{\Gamma\left(g_{Y} \Delta\right)}{\Gamma\left(g_{Y} \Delta+\frac{1}{2}\right)} .
$$

It is easy to see that $\rho(x, y)$ satisfies the $(2+1)$-dimensional massless Dirac equation with the Dirac algebra Eq. (8).

Thus we see that two-component Dirac fermions in $(2+1)$ dimensions are relevant for the dynamics of zero modes localized at the domain walls. Indeed, recently [9] it has been suggested that the remarkable phenomenon of the spontaneous generation of uniform magnetic condensate in $(2+1)$-dimensional $Q E D$ gives rise to ferromagnetic domain walls at the cosmological electroweak phase transition which could account for the cosmological primordial 
magnetic field.

The aim of this paper is to study in details the $(2+1)$-dimensional $Q E D$ with two-component (parity-violating) and four-component (parity-invariant) fermions both at zero and finite temperature. In particular we compare in details the $(2+1)$-dimensional $Q E D$ coupled with both two and four components Dirac fermions in the one-loop approximations. For pedagogical reasons, we present our results in greater details with complete calculations which are not available in our previuos published papers. The plan of the paper is as follows. In Sect. 2 we consider Dirac fermions with P-odd mass term (two component fermions) at zero and finite temperature. Section 3 is devoted to the study of P-even Dirac fermions. In Sect. 4 we discuss the spontaneous generation of a constant fermion mass triggered by an external magnetic field. Finally our conclusions are drawn in Sect. 5.

\section{P-Odd Fermions}

In this section we discuss relativistic fermions coupled with the electromagnetic field in $(2+1)$-dimensions. The relevant Lagrangian is:

$$
\mathcal{L}_{Q E D}=-\frac{1}{4} F_{\mu \nu}(x) F^{\mu \nu}(x)+\bar{\psi}(x)[i \not \partial-e A(x)-m] \psi(x) .
$$

From the Lagrangian Eq. (12) it is easy to obtain the equations of motion. In particular the fermion fields satisfy the familiar Dirac equation :

$$
\left(i \gamma^{\mu} \partial_{\mu}-e \gamma^{\mu} A_{\mu}-m\right) \psi=0
$$

The gamma matrices satisfy the Clifford algebra :

$$
\left\{\gamma^{\mu}, \gamma^{\nu}\right\}=2 g^{\mu \nu}, \quad \gamma^{\mu} \gamma^{\nu}=g^{\mu \nu}-i \epsilon^{\mu \nu \rho} \gamma_{\rho}, \quad \gamma_{\mu}=g_{\mu \nu} \gamma^{\nu}
$$

with the flat Minkowski metric is $g^{\mu \nu}=\operatorname{diag}(1,-1,-1)$.

A spinorial representation in three dimensions is provided by two-component Dirac spinors. The fundamental representation of the Clifford algebra is given by $2 \times 2$ matrices which can be constructed from the Pauli matrices: 


$$
\gamma^{0}=\sigma_{3}, \quad \gamma^{1}=i \sigma_{1}, \quad \gamma^{2}=i \sigma_{2}
$$

We can define the parity and time-reversal transformation [1]

$$
\begin{aligned}
& \mathcal{P} A^{0}(\vec{x}, t) \mathcal{P}^{-1}=A^{0}\left(\overrightarrow{x^{\prime}}, t\right) \\
& \mathcal{P} A^{1}(\vec{x}, t) \mathcal{P}^{-1}=-A^{1}\left(\overrightarrow{x^{\prime}}, t\right) \\
& \mathcal{P} A^{2}(\vec{x}, t) \mathcal{P}^{-1}=A^{2}\left(\overrightarrow{x^{\prime}}, t\right) \\
& \mathcal{P} \psi(\vec{x}, t) \mathcal{P}^{-1}=\sigma_{1} \psi\left(\overrightarrow{x^{\prime}}, t\right)
\end{aligned}
$$

with $\vec{x}=\left(x_{1}, x_{2}\right)$ and $\overrightarrow{x^{\prime}}=\left(-x_{1}, x_{2}\right)$,

$$
\begin{aligned}
& \mathcal{T} A^{0}(\vec{x}, t) \mathcal{T}^{-1}=A^{0}(\vec{x},-t) \\
& \mathcal{T} \vec{A}(\vec{x}, t) \mathcal{T}^{-1}=-\vec{A}(\vec{x},-t) \\
& \mathcal{T} \psi(\vec{x}, t) \mathcal{T}^{-1}=\sigma_{2} \psi(\vec{x},-t) .
\end{aligned}
$$

Note that in this representation the mass term $m \bar{\psi} \psi$ is odd under both $\mathcal{P}$ and $\mathcal{T}$ transformations [1]. It is important to point out that this parity-violating mass is, in fact, the only possibility in the two-component formalism.

Let us consider, now, the Hamiltonian in the fixed-time temporal gauge $A_{0}=0$ :

$$
\begin{aligned}
H=\int d^{2} x\left\{\frac{1}{2} \vec{E}^{2}(x)+\frac{1}{2} B^{2}(x)+\psi^{\dagger}(x)[-i \vec{\alpha} \cdot \vec{\nabla}+\beta m] \psi(x)+\right. \\
\left.-e \psi^{\dagger}(x) \vec{\alpha} \cdot \vec{A}(x) \psi(x)\right\}
\end{aligned}
$$

where $\beta=\gamma^{0}$ and $\vec{\alpha}=\gamma^{0} \vec{\gamma}$.

Note that in two spatial dimensions the magnetic field is a (pseudo-)scalar. We are interested in the case of spinorial quantum electrodynamics in presence of a background field. So that we write :

$$
A_{k}(x)=\bar{A}_{k}(x)+\eta_{k}(x)
$$

where $\eta(x)$ is the fluctuation over the background $\bar{A}(x)$.

We consider a background field which corresponds to an uniform magnetic field. Thus in the so-called Landau gauge we have : 


$$
\bar{A}_{k}(x)=\delta_{k 2} x_{1} B \quad k=1,2 .
$$

Inserting Eq. (19) into Eq. (18), in the one loop approximation we rewrite the Hamiltonian as :

$$
\begin{aligned}
H_{0}= & H_{\eta}+H_{D}+V \frac{B^{2}}{2}=\int d^{2} x\left\{\frac{1}{2} \vec{E}^{2}(x)+\frac{1}{2}\left[\epsilon_{i j} \partial_{i} \eta_{j}(x)\right]^{2}\right\}+ \\
& +\int d^{2} x\left\{\psi^{\dagger}(x)\left[\alpha_{k}\left(-i \partial_{k}-e \bar{A}_{k}\right)+\beta m\right] \psi(x)\right\}+V \frac{B^{2}}{2}
\end{aligned}
$$

where $V$ is the spatial volume. In this approximation the Dirac equation reduces to:

$$
i \frac{\partial \psi(\vec{x}, t)}{\partial t}=[\vec{\alpha} \cdot(-i \vec{\nabla}-e \vec{A})+\beta m] \psi(\vec{x}, t) .
$$

As it is well known the Dirac equation Eq. (22) can be exactly solved [13]. Indeed, taking

$$
\psi(\vec{x}, t)=e^{-i E t} \psi(\vec{x}),
$$

we get :

$$
[\vec{\alpha} \cdot(-i \vec{\nabla}-e \vec{A})+\beta m] \psi(\vec{x})=E \psi(\vec{x}) .
$$

It is straightforward to obtain the solutions of Eq. (23) (we assume $e B>0$ ) :

$$
\begin{gathered}
E=+E_{n}, n \geq 1 \psi_{n, p}^{(+)}=\sqrt{\frac{E_{n}+m}{2 E_{n}}} \frac{e^{i p x_{2}}}{\sqrt{2 \pi}} e^{-\frac{1}{2} \zeta^{2}}\left(\begin{array}{c}
N_{n} H_{n}(\zeta) \\
-\frac{\sqrt{E_{n}^{2}-m^{2}}}{E_{n}+m} N_{n-1} H_{n-1}(\zeta)
\end{array}\right) \\
E=-E_{n}, n \geq 1 \psi_{n, p}^{(-)}=\sqrt{\frac{E_{n}-m}{2 E_{n}}} \frac{e^{i p x_{2}}}{\sqrt{2 \pi}} e^{-\frac{1}{2} \zeta^{2}}\left(\begin{array}{c}
N_{n} H_{n}(\zeta) \\
-\frac{\sqrt{E_{n}^{2}-m^{2}}}{E_{n}-m} N_{n-1} H_{n-1}(\zeta)
\end{array}\right) \\
E=E_{0}=m \quad \psi_{0, p}=N_{0} \frac{e^{i p x_{2}}}{\sqrt{2 \pi}} e^{-\frac{1}{2} \zeta^{2}}\left(\begin{array}{c}
1 \\
0
\end{array}\right),
\end{gathered}
$$

where the energy spectrum is :

$$
E_{n}=\sqrt{2 n e B+m^{2}}
$$


and

$$
N_{n}=\frac{\left(\frac{e B}{\pi}\right)^{\frac{1}{4}}}{\sqrt{2^{n} n !}}, \quad \zeta=\sqrt{e B}\left(x_{1}-\frac{p}{e B}\right)
$$

$H_{n}(x)$ is the $n$-th Hermite polynomial, $\psi_{n, p}^{(+)}$and $\psi_{n, p}^{(-)}$are respectively positive and negative energy solutions which are normalized as:

$$
\int_{-\infty}^{+\infty} d^{2} x \psi_{n, p}^{( \pm)^{\dagger}}(\vec{x}) \psi_{n^{\prime}, p^{\prime}}^{( \pm)}(\vec{x})=\delta\left(p-p^{\prime}\right) \delta_{n n^{\prime}}
$$

Note that due to the zero modes Eq. (24c) the spectrum is not charge symmetric (see Fig. 1). The Landau levels are infinitely degenerate with density of states:

$$
\int_{-\infty}^{+\infty} d p \psi_{n, p}^{( \pm)^{\dagger}}(\vec{x}) \psi_{n, p}^{( \pm)}(\vec{x})=\frac{e B}{2 \pi} .
$$

It is convenient to expand the fermion field operator $\psi(\vec{x})$ in terms of the wave function basis $\psi_{n, p}^{(+)}$and $\psi_{n, p}^{(-)}$. In other words, we adopt the so-called Furry's representation. We have:

$$
\begin{aligned}
& m<0 \quad \psi(x)=\sum_{n=1}^{\infty} \int_{-\infty}^{+\infty} d p a_{n p} \psi_{n p}^{(+)}(x)+\sum_{n=0}^{\infty} \int_{-\infty}^{+\infty} d p b_{n p}^{\dagger} \psi_{n p}^{(-)} \\
& m>0 \quad \psi(x)=\sum_{n=0}^{\infty} \int_{-\infty}^{+\infty} d p a_{n p} \psi_{n p}^{(+)}(x)+\sum_{n=1}^{\infty} \int_{-\infty}^{+\infty} d p b_{n p}^{\dagger} \psi_{n p}^{(-)} .
\end{aligned}
$$

We observe that in the case of positive mass the positive solutions have eigenvalues $+E_{n}$ with $n \geq 0$ and negative ones $-E_{n}$ with $n \geq 1$ and vice versa in the case of negative mass. In the expansion of $\psi$ we associate particle operators to positive energy solutions and antiparticle operators to negative energy solutions. These operators satisfy the standard anticommutation relations:

$$
\left\{a_{i}(n, p), a_{j}^{\dagger}\left(n^{\prime}, p^{\prime}\right)\right\}=\left\{b_{i}(n, p), b_{j}^{\dagger}\left(n^{\prime}, p^{\prime}\right)\right\}=\delta_{i j} \delta_{n n^{\prime}} \delta\left(p-p^{\prime}\right)
$$

the others anticommutators being zero. The Dirac Hamiltonian operator

$$
H_{D}=\int d^{2} x\left[\psi^{\dagger}(x)(-i \vec{\alpha} \cdot \vec{\nabla}-\vec{\alpha} \cdot \overrightarrow{\bar{A}}+\beta m) \psi(x)\right]
$$

in the Furry's representation reads: 


$$
\begin{aligned}
& m>0 \quad H_{D}=\int_{-\infty}^{+\infty} d p\left[\sum_{n=0}^{\infty} E_{n} a_{n p}^{\dagger} a_{n p}+\sum_{n=1}^{\infty} E_{n} b_{n p}^{\dagger} b_{n p}\right]-\frac{e B}{2 \pi} V \sum_{n=1}^{\infty} E_{n} \\
& m<0 \quad H_{D}=\int_{-\infty}^{+\infty} d p\left[\sum_{n=1}^{\infty} E_{n} a_{n p}^{\dagger} a_{n p}+\sum_{n=0}^{\infty} E_{n} b_{n p}^{\dagger} b_{n p}\right]-\frac{e B}{2 \pi} V \sum_{n=0}^{\infty} E_{n} .
\end{aligned}
$$

Let us, firstly, evaluate the fermion condensate at zero temperature. By using the expansions Eqs. (29) and (30), it is straightforward to obtain:

$$
m>0 \quad\langle 0|\bar{\psi} \psi| 0\rangle=-\frac{m e B}{2 \pi} \sum_{n=1}^{\infty} \frac{1}{E_{n}}
$$

and

$$
m<0 \quad\langle 0|\bar{\psi} \psi| 0\rangle=-\frac{m e B}{2 \pi} \sum_{n=0}^{\infty} \frac{1}{E_{n}} .
$$

In order to evaluate the massless limit, we observe that

$$
\sum_{n=1}^{\infty} \frac{1}{E_{n}}=\frac{1}{\sqrt{2 e B}} \sum_{n=1}^{\infty} \frac{1}{\sqrt{n+x}}
$$

where $x=\frac{m^{2}}{2 e B}$. We recognize in Eq. (37) the Hurwitz zeta function that in the massless limit $(x \rightarrow 0)$ reduces to the Riemann zeta function $\zeta\left(\frac{1}{2}\right) \simeq-1.46035451$. So that in the massless limit we get [7]:

$$
\lim _{m \rightarrow 0^{-}}\langle 0|\bar{\psi} \psi| 0\rangle=\frac{e B}{2 \pi}
$$

and

$$
\lim _{m \rightarrow 0^{+}}\langle 0|\bar{\psi} \psi| 0\rangle=0
$$

Therefore we see that in the massless limit the magnetic field gives origin to a fermion condensate only in the case of a vanishing negative mass term.

We consider, now, the background field effective action. As it is well known, in the one-loop approximation the background field effective action coincides with the vacuum energy in presence of the static background field. In our case we need to evaluate the vacuum energy density in presence of the constant magnetic field $B$.

To this end we need to evaluate the Dirac energy, $E_{D}$, defined as : 


$$
E_{D}=\frac{\left\langle 0\left|H_{D}\right| 0\right\rangle}{\langle 0 \mid 0\rangle}
$$

where $|0\rangle$ is the fermion vacuum. By using Eqs. (33), (34), it is straightforward to obtain the Dirac energy:

$$
m<0 \quad E_{D}(B)=-\frac{e B}{2 \pi} V \sum_{n=0}^{\infty} E_{n}
$$

and

$$
m>0 \quad E_{D}(B)=-\frac{e B}{2 \pi} V \sum_{n=1}^{\infty} E_{n} .
$$

Note that Eqs. (41) and (42) differ in the term $|m| \frac{e B}{2 \pi} V$.

To perform the sums in Eqs. (41) and (42) we use the integral representation :

$$
\sqrt{a}=-\int_{0}^{\infty} \frac{d s}{\sqrt{\pi s}} \frac{d}{d s} e^{-a s} .
$$

We have :

$$
-\frac{e B}{2 \pi} V \sum_{n=0}^{\infty} E_{n}=\frac{e B}{2 \pi} V \int_{0}^{\infty} \frac{d s}{\sqrt{\pi s}} \frac{d}{d s}\left(\frac{e^{-m^{2} s}}{1-e^{-2 e B s}}\right)
$$

Let us consider the negative mass case. By using Eq. (44) we rewrite Eq. (41) as follows :

$$
E_{D}(B)=\frac{e B}{2 \pi} V \int_{0}^{\infty} \frac{d s}{\sqrt{\pi s}} \frac{d}{d s}\left(\frac{e^{-m^{2} s}}{1-e^{-2 e B s}}\right)
$$

whence, after subtracting $E_{D}(B=0)$,

$$
E_{D}(B)-E_{D}(B=0)=\frac{e B}{2 \pi} V \int_{0}^{\infty} \frac{d s}{\sqrt{\pi s}} \frac{d}{d s}\left(\frac{e^{-m^{2} s}}{1-e^{-2 e B s}}-\frac{e^{-m^{2} s}}{2 e B s}\right) .
$$

Introducing the dimensionless variable $\lambda=\frac{e B}{m^{2}}$ and the function

$$
g(\lambda)=\int_{0}^{\infty} \frac{d s}{\sqrt{\pi s}} \frac{d}{d s}\left[\frac{e^{-\frac{s}{\lambda}}}{1-e^{-2 s}}-\frac{e^{-\frac{s}{\lambda}}}{2 s}\right]
$$

we obtain the vacuum energy density $\mathcal{E}=\frac{E}{V}$ :

$$
\tilde{\mathcal{E}}_{m<0}=\mathcal{E}_{m<0}(B)-\mathcal{E}(0)=\frac{B^{2}}{2}+\frac{(e B)^{\frac{3}{2}}}{2 \pi} g\left(\frac{e B}{m^{2}}\right),
$$


where we added the classical magnetic energy density.

The case of positive mass can be handled in the same way. We get :

$$
\tilde{\mathcal{E}}_{m>0}=\mathcal{E}_{m>0}(B)-\mathcal{E}(0)=\frac{B^{2}}{2}+\frac{(e B)^{\frac{3}{2}}}{2 \pi} g\left(\frac{e B}{m^{2}}\right)+\frac{e B}{2 \pi}|m| .
$$

We introduce the further dimensionless parameter $\alpha=\frac{|m|}{e^{2}}$ to rewrite Eqs. (48) and (49) as :

$$
\begin{gathered}
\frac{\tilde{\mathcal{E}}_{m<0}}{|m|^{3}}=\frac{\lambda^{2} \alpha}{2}+\frac{\lambda^{\frac{3}{2}}}{2 \pi} g(\lambda), \\
\frac{\tilde{\mathcal{E}}_{m>0}}{|m|^{3}}=\frac{\lambda^{2} \alpha}{2}+\frac{\lambda^{\frac{3}{2}}}{2 \pi} g(\lambda)+\frac{\lambda}{2 \pi} .
\end{gathered}
$$

In Figures 2 and 3 we plot Eq. (50) and (51) respectively as a function of $\lambda$ for two different values of $\alpha$.

As it is evident the background field effective potential displays a negative minimum only in the case of fermions with negative mass term [5]. Indeed, using the expansion :

$$
g(\lambda) \stackrel{\lambda \rightarrow 0}{\sim}-\frac{1}{2 \lambda^{\frac{1}{2}}}+\frac{\lambda^{\frac{1}{2}}}{12},
$$

we have :

$$
\tilde{\mathcal{E}}_{m<0} \sim-\frac{e B}{4 \pi}|m|+\frac{1}{24 \pi} \frac{(e B)^{2}}{|m|}+\frac{B^{2}}{2} .
$$

We see that the negative linear term, which is absent in the positive mass case, is responsible of the negative minimum in vacuum energy density.

It is worthwhile to evaluate the thermal corrections to the condensate and to the vacuum energy density. Our approximation corresponds to perform the thermal average with respect to the one-loop Hamiltonian $H_{0}$, Eqs. (21). We introduce the symbol $\langle\ldots\rangle_{\beta}$ to mean the thermal average with respect to the Hamiltonian $H_{0}$. Let us observe that $\left\langle a_{n p}^{\dagger} a_{n p}\right\rangle_{\beta}$ and $\left\langle b_{n p}^{\dagger} b_{n p}\right\rangle_{\beta}$ can be expressed in terms of the equilibrium occupation number $n(p)$ :

$$
\begin{gathered}
n(p)=\left\langle a_{n p}^{\dagger} a_{n p}\right\rangle_{\beta}=1-\left\langle a_{n p} a_{n p}^{\dagger}\right\rangle_{\beta}=\frac{1}{e^{\beta E_{n}}+1}, \\
1-n(p)=\left\langle b_{n p} b_{n p}^{\dagger}\right\rangle_{\beta}=1-\left\langle b_{n p}^{\dagger} b_{n p}\right\rangle_{\beta}=\frac{1}{e^{-\beta E_{n}}+1} .
\end{gathered}
$$


It is, now, easy to evaluate the fermion condensate at finite temperature. We have for $m<0$ :

$$
\langle\bar{\psi} \psi\rangle_{\beta}=\sum_{n=1}^{\infty} \int d p\left\langle a_{n p}^{\dagger} a_{n p}\right\rangle_{\beta} \psi_{n, p}^{(+)} \gamma^{\dagger} \gamma^{0} \psi_{n, p}^{(+)}+\sum_{n=0}^{\infty} \int d p\left\langle b_{n p} b^{\dagger}{ }_{n p}\right\rangle_{\beta} \psi_{n, p}^{(-)}{ }^{\dagger} \gamma^{0} \psi_{n, p}^{(-)},
$$

with $\psi_{n, p}^{(+)}$and $\psi_{n, p}^{(-)}$given by Eqs. (24a,b,c).

Using Eq. (54) and Eq. (55) we obtain the fermion condensate at finite temperature:

$$
\langle\bar{\psi} \psi\rangle_{\beta}=\frac{e B}{2 \pi} \frac{1}{e^{\beta m}+1}-\frac{m e B}{2 \pi} \sum_{n=1}^{\infty} \frac{1}{E_{n}} \tanh \left(\frac{\beta}{2} E_{n}\right) .
$$

One can check that this last expression holds for both positive and negative mass.

If we perform the massless limit, then we face with the problem of the order of the limits $m \rightarrow 0$ and $\beta \rightarrow \infty$. Indeed we have:

$$
\lim _{m \rightarrow 0^{+}} \lim _{\beta \rightarrow \infty}\langle\bar{\psi} \psi\rangle_{\beta}=0 \quad \lim _{m \rightarrow 0^{-}} \lim _{\beta \rightarrow \infty}\langle\bar{\psi} \psi\rangle_{\beta}=\frac{e B}{2 \pi},
$$

while

$$
\lim _{\beta \rightarrow \infty} \lim _{m \rightarrow 0^{-}}\langle\bar{\psi} \psi\rangle_{\beta}=\lim _{\beta \rightarrow \infty} \lim _{m \rightarrow 0^{+}}\langle\bar{\psi} \psi\rangle_{\beta}=\frac{e B}{4 \pi} .
$$

Of course Eq. (58) agrees with the zero temperature result. On the other hand, Eq. (59) shows that the massless limit of the fermion condensate at any temperature is T-independent and symmetric in $m$. Thus, the fermion condensate $\langle\bar{\psi} \psi\rangle_{\beta}$ is a non-analytic function at the origin in the $(m, T)$-plane and its limit value depends on the order of the zero-mass and $\beta$-infinity limit. This non-analitycity of the thermal condensate is also present in the P-invariant formulation of the theory [14], as we will see in the next Section. A similar nonanalyticity has been noticed by the authors of Ref. 15] in the study of induced quantum numbers at finite temperature. It is worthwhile to stress that the presence of a heat bath, even with an infinitesimal temperature, makes the massless limit of the condensate symmetric and different from zero. Moreover, from Eq. (59) we see that the fermion condensate of the zero mass theory in presence of an external constant magnetic field corresponds to half filled zero modes [11].

Let us turn on the finite temperature effective potential. As it is well known, the relevant quantity is the free energy density. We have : 


$$
F=-\frac{1}{\beta} \ln Z
$$

where $Z$ is the partition function

$$
Z=\operatorname{Tr}\left(e^{-\beta H}\right)
$$

In the one-loop approximation we get :

$$
F_{0}=-\frac{1}{\beta} \ln Z_{0}, \quad Z_{0}=\operatorname{Tr}\left[e^{-\beta H_{0}}\right]
$$

In our approximation the free energy is the sum of the photonic and fermionic contributions. Only the latter depends on the magnetic field. So that the calculation of the free energy reduces to consider the partition function of relativistic fermions in presence of the magnetic field.

We consider the case $m>0$. A standard calculation gives :

$$
Z_{0}=\operatorname{Tr} e^{-\beta\left(H_{D}+\frac{V B^{2}}{2}\right)}=e^{-\beta \frac{V B^{2}}{2}}\left(1+e^{-\beta E_{0}}\right)^{N_{d}} \prod_{n=1}^{\infty}\left(1+e^{-\beta E_{n}}\right)^{2 N_{d}} e^{\beta E_{n} N_{d}}
$$

where $N_{d}$ is the degeneracy of the Landau levels:

$$
N_{d}=\frac{e B}{2 \pi} V
$$

The free energy density $\mathcal{F}_{0}(B)=\frac{F_{0}(B)}{V}$ is :

$$
\mathcal{F}_{0}^{m>0}(B)=-\frac{e B}{\pi \beta} \sum_{n=1}^{\infty} \ln \left(1+e^{-\beta E_{n}}\right)-\frac{e B}{2 \pi \beta} \ln \left(1+e^{-\beta E_{0}}\right)-\frac{e B}{2 \pi} \sum_{n=1}^{\infty} E_{n}+\frac{B^{2}}{2} .
$$

In the same way we obtain :

$$
\mathcal{F}_{0}^{m<0}(B)=-\frac{e B}{\pi \beta} \sum_{n=1}^{\infty} \ln \left(1+e^{-\beta E_{n}}\right)-\frac{e B}{2 \pi \beta} \ln \left(1+e^{-\beta E_{0}}\right)-\frac{e B}{2 \pi} \sum_{n=0}^{\infty} E_{n}+\frac{B^{2}}{2} .
$$

In Eqs. (65) and (66) the first term on the right hand side arises from the negative and positive Landau levels with $n>0$, the second one is the zero mode contribution, while the last two terms correspond to the zero temperature vacuum energy density. To calculate the sums in Eqs. (65) and (66) we expand the logarithm: 


$$
I_{1}(B)=-\frac{e B}{\pi \beta} \sum_{n=1}^{\infty} \ln \left(1+e^{-\beta E_{n}}\right)=-\frac{e B}{\pi \beta} \sum_{n=1}^{\infty} \sum_{k=1}^{\infty} \frac{(-1)^{k+1}}{k} e^{-\beta k E_{n}}
$$

and use the integral representation [16] :

$$
e^{-\sqrt{a b}}=2 \sqrt{\frac{a}{\pi}} \int_{0}^{\infty} e^{-a x^{2}-\frac{b}{x^{2}}} d x \quad a>0, \quad b>0 .
$$

This allows us to evaluate the sum over $n$ :

$$
\sum_{n=1}^{\infty} e^{-\beta k E_{n}}=\frac{1}{\sqrt{\pi}} \int_{0}^{\infty} e^{-\frac{x^{2}}{4}-\frac{1}{x^{2}} \beta^{2} k^{2} m^{2}} \frac{1}{e^{\frac{2 \beta^{2} e B k^{2}}{x^{2}}}-1}
$$

Therefore we have :

$$
I_{1}(B)=-\frac{e B}{\pi^{\frac{3}{2}} \beta} \sum_{k=1}^{\infty} \frac{(-1)^{k+1}}{k} \int_{0}^{\infty} d x e^{-\frac{x^{2}}{4}-\frac{1}{x^{2}} \beta^{2} k^{2} m^{2}} \frac{1}{e^{\frac{2 \beta^{2} e B k^{2}}{x^{2}}}-1}
$$

After subtracting the contribution at $B=0$ we obtain :

$$
\begin{aligned}
\tilde{I}_{1}(B) & =I_{1}(B)-I_{1}(B=0)= \\
& =-\frac{e B}{\pi^{\frac{3}{2}} \beta} \sum_{k=1}^{\infty} \frac{(-1)^{k+1}}{k} \int_{0}^{\infty} d x e^{-\frac{x^{2}}{4}-\frac{1}{x^{2}} \beta^{2} k^{2} m^{2}}\left[\frac{1}{e^{\frac{2 \beta^{2} e B k^{2}}{x^{2}}}-1}-\frac{x^{2}}{2 e B \beta^{2} k^{2}}\right] .
\end{aligned}
$$

The sum over $k$ is rapidly convergent. So that this last expression is amenable to numerical evaluations. Putting it all together we finally get :

$$
\begin{aligned}
\tilde{\mathcal{F}}_{0}^{m>0}(B) & =\mathcal{F}_{0}^{m>0}(B)-\mathcal{F}_{0}^{m>0}(B=0)= \\
& =\tilde{I}_{1}(B)-\frac{e B}{2 \pi \beta} \ln \left(1+e^{-\beta E_{0}}\right)+\tilde{\mathcal{E}}_{m>0}(B)
\end{aligned}
$$

and

$$
\begin{aligned}
\tilde{\mathcal{F}}_{0}^{m<0}(B) & =\mathcal{F}_{0}^{m<0}(B)-\mathcal{F}_{0}^{m<0}(B=0)= \\
& =\tilde{I}_{1}(B)-\frac{e B}{2 \pi \beta} \ln \left(1+e^{-\beta E_{0}}\right)+\tilde{\mathcal{E}}_{m<0}(B)
\end{aligned}
$$

In Figures 4 and 5 we display the free energy density, respectively Eq. (72) and Eq. (73), for different temperatures $\hat{T}=\frac{T}{|m|}$ and $\alpha=\frac{|m|}{e^{2}}=0.1$, as a function of $\lambda=\frac{e B}{m^{2}}$.

It is remarkable to observe that in the case $m<0$ by increasing the temperature the negative minimum does not disappear. This means that at high temperature there is not restoration 
of the symmetry broken at zero temperature. In other words, the spontaneous generation of the magnetic condensate survives even at infinite temperature. Remarkably enough, the authors of Ref. [10], within a different approach, found similar results also in presence of the Chern-Simon term .

It is interesting to study the high temperature asymptotic expansion of the free energy density in the case of negative mass. Indeed we have :

$$
I_{1}(B)=\frac{e B}{2 \pi \beta} \ln 2+\frac{e B}{2 \pi} \sum_{n=1}^{\infty} E_{n}+\mathcal{O}\left(\beta^{2}\right)
$$

moreover

$$
-\frac{e B}{2 \pi \beta} \ln \left(1+e^{-\beta E_{0}}\right)=-\frac{e B}{2 \pi \beta} \ln 2+\frac{e B}{4 \pi}|m|+\mathcal{O}\left(\beta^{2}\right) .
$$

So that:

$$
\tilde{\mathcal{F}}_{0}^{m<0}(B)=-\frac{e B}{4 \pi}|m|+\frac{B^{2}}{2}+\mathcal{O}\left(\beta^{2}\right)
$$

We see that the linear term in $|m|$ gives rise to the negative minimum at any finite temperature. Moreover, the slope of the linear term coincides with the one at zero temperature. This is clearly seen in Fig. 6 where we display the free energy density without the classical energy $\frac{B^{2}}{2}$ for three different values of the temperature.

The negative minimum of Eq. (76) is at

$$
e B^{*}=\frac{e^{2}|m|}{4 \pi}
$$

As a consequence the negative condensation energy is :

$$
\tilde{\mathcal{F}}_{0}^{m<0}\left(B^{*}\right)=-\frac{e B^{*}}{2}|m|=-\frac{e^{2}|m|^{2}}{32 \pi^{2}}
$$

The minimum of Eq. (77) coincides with the zero temperature minimum in the "weakcoupling" region $\alpha>>1$ :

$$
\frac{e B^{*}}{m^{2}}=\frac{1}{4 \pi \alpha}
$$


We would like to conclude this Section by stressing that our results show that the negative minimum in the free energy density is due to the term linear in the magnetic field. This term, in turn, is accounted for by the contributions due to the zero modes, and it is present only for negative fermion mass. Moreover, we find that the thermal corrections to the above mentioned coefficient are small and vanish at infinite temperature. For these reasons we feel that higher order corrections do not should modify the spontaneous generation of the magnetic condensate .

\section{P-Even Fermions}

In three dimensions we have a realization of the Dirac algebra which is different from the $2 \times 2$ representation Eq. (15). In this representation the Dirac fermions are four-component spinor and the three $4 \times 4 \gamma$-matrices can be taken to be [17:

$$
\gamma^{0}=\left(\begin{array}{cc}
\sigma_{3} & 0 \\
0 & -\sigma_{3}
\end{array}\right) \quad \gamma^{1}=\left(\begin{array}{cc}
i \sigma_{1} & 0 \\
0 & -i \sigma_{1}
\end{array}\right) \quad \gamma^{2}=\left(\begin{array}{cc}
i \sigma_{2} & 0 \\
0 & -i \sigma_{2}
\end{array}\right)
$$

The representation given by Eq. (80) corresponds to a reducible representation of the Dirac algebra. In this representation the fermionic mass term $m \bar{\psi} \psi$ is parity conserving. Indeed, comparing Eq. (15) with Eq. (80) we see that a four-component fermion with mass $m$ corresponds to two two-component fermions with mass $m$ amd $-m$ respectively.

As the first step, we need to solve the Dirac equation Eq. (23) with the Dirac algebra representation Eq. (80). Indeed, it is straightforward to obtain the orthonormal positive and negative energy solutions of Eq. (23). We get :

$$
E=+E_{n}, n \geq 1 \psi_{1}^{(+)}=\sqrt{\frac{E_{n}+m}{2 E_{n}}} e^{i p x_{2}-\frac{1}{2} \zeta^{2}}\left(\begin{array}{c}
N_{n} H_{n}(\zeta) \\
-\frac{\sqrt{E_{n}^{2}-m^{2}}}{E_{n}+m} N_{n-1} H_{n-1}(\zeta) \\
0 \\
0
\end{array}\right)
$$




$$
\begin{aligned}
& E=+E_{n}, n \geq 1 \psi_{2}^{(+)}=\sqrt{\frac{E_{n}+m}{2 E_{n}}} e^{i p x_{2}-\frac{1}{2} \zeta^{2}}\left(\begin{array}{c}
0 \\
0 \\
-\frac{\sqrt{E_{n}^{2}-m^{2}}}{E_{n}+m} N_{n} H_{n}(\zeta) \\
N_{n-1} H_{n-1}(\zeta)
\end{array}\right) \\
& E=-E_{n}, n \geq 1 \psi_{1}^{(-)}=\sqrt{\frac{E_{n}+m}{2 E_{n}}} e^{-i p x_{2}-\frac{1}{2} \xi^{2}}\left(\begin{array}{c}
\frac{\sqrt{E_{n}^{2}-m^{2}}}{E_{n}+m} N_{n} H_{n}(\xi) \\
N_{n-1} H_{n-1}(\xi) \\
0 \\
0
\end{array}\right) \\
& E=-E_{n}, n \geq 1 \psi_{2}^{(-)}=\sqrt{\frac{E_{n}+m}{2 E_{n}}} e^{-i p x_{2}-\frac{1}{2} \xi^{2}}\left(\begin{array}{c}
0 \\
0 \\
N_{n} H_{n}(\xi) \\
\frac{\sqrt{E_{n}^{2}-m^{2}}}{E_{n}+m} N_{n-1} H_{n-1}(\xi)
\end{array}\right)
\end{aligned}
$$

with the same notations as in Eqs. (25), (26) and $\xi=\sqrt{e B}\left(x_{1}+\frac{p}{e B}\right)$. The zero mode wave functions depend on sign of the mass term. If $m>0$ we have:

$$
\begin{aligned}
& E_{0}=|m| \quad \psi_{0, m>0}^{(+)}=N_{0} e^{i p x_{2}-\frac{1}{2} \zeta^{2}}\left(\begin{array}{l}
1 \\
0 \\
0 \\
0
\end{array}\right) \\
& E_{0}=|m| \quad \psi_{0, m>0}^{(-)}=N_{0} e^{i p x_{2}-\frac{1}{2} \xi^{2}}\left(\begin{array}{l}
0 \\
0 \\
1 \\
0
\end{array}\right) .
\end{aligned}
$$

For $m<0$ the zero mode wave functions are obtained by $\psi_{0, m<0}^{ \pm}=\gamma^{5} \psi_{0, m>0}^{ \pm}$, where: 


$$
\gamma^{5}=i\left(\begin{array}{rr}
0 & I \\
-I & 0
\end{array}\right)
$$

We stress that the density of the states for the zero modes is $\frac{|e B|}{2 \pi}$ while it is $\frac{|e B|}{\pi}$ for the modes with $n \geq 1$. As already noticed, this is due to the fact that for $n \geq 1$ a four-component fermion corresponds to two independent degenerate two-component fermions.

As in the previous section to calculate the condensate at $T=0$, we expand the field operator on the basis of the wave functions Eqs. (81a-d), (82a,b):

$$
\psi=\int_{-\infty}^{+\infty} \frac{d p}{\sqrt{2 \pi}}\left[a(0, p) \psi_{0, p}^{(+)}+b^{\dagger}(0, p) \psi_{0, p}^{(-)}\right]+\sum_{n=1}^{\infty} \sum_{i=1,2} \int_{-\infty}^{+\infty} \frac{d p}{\sqrt{2 \pi}}\left[a_{i}(n, p) \psi_{i, n, p}^{(+)}+b_{i}^{\dagger}(n, p) \psi_{i, n, p}^{(-)}\right]
$$

where the operators $a_{i}$ and $b_{i}$ satisfy the anticommutation relations Eq. (31).

It is possible to obtain the fermion condensate at $T=0$ through the propagator $G(x, y)=$ $\langle 0|T \psi(x) \bar{\psi}(y)| 0\rangle:$

$$
\langle 0|\bar{\psi} \psi| 0\rangle=-\lim _{x \rightarrow y} \operatorname{tr} G(x, y)
$$

by using the Schwinger proper time approach [18]. However, in the Furry's representation and in the one-loop approximation it is rather simple to obtain the condensate at $T=0$ :

$$
\langle 0|\bar{\psi} \psi| 0\rangle=-\frac{m}{|m|} \frac{e B}{2 \pi}-\frac{m e B}{\pi} \sum_{n=1}^{\infty} \frac{1}{E_{n}}
$$

The massless limit of the condensate reads :

$$
\left.\langle 0|\bar{\psi} \psi| 0\rangle\right|_{m \rightarrow 0}=-\frac{m}{|m|} \frac{e B}{2 \pi} .
$$

Although the massless limit of the fermion condensate is non-vanishing, it depends on the sign of $m$. This means that the limit value does depend on the limiting procedure. For instance, if one performs that symmetric massless limit, then one gets a vanishing condensate. As a matter of fact, we shall show below that at any finite temperature the fermion condensate vanishes for $m \rightarrow 0$. To see that, we evaluate the thermal correction to the 
condensate. In our approximation and taking into account Eqs. (54), (55) and (84), it is straightforward to calculate the finite temperature fermion condensate. We get:

$$
\langle\bar{\psi} \psi\rangle_{\beta}=-\frac{m}{|m|} \frac{e B}{2 \pi} \tanh \left(\frac{\beta}{2} E_{0}\right)-\frac{m e B}{\pi} \sum_{n=1}^{\infty} \frac{1}{E_{n}} \tanh \left(\frac{\beta}{2} E_{n}\right) .
$$

This expression is in agreement with Ref. [14], where the authors calculate the value of the condensate at finite temperature using the thermo field dynamics [19] approach with a non-vanishing chemical potential.

Let us consider the limits $m \rightarrow 0$ and $\beta \rightarrow \infty$. We have:

$$
\lim _{m \rightarrow 0} \lim _{\beta \rightarrow \infty}\langle\bar{\psi} \psi\rangle_{\beta}=-\frac{m}{|m|} \frac{e B}{2 \pi},
$$

which, of course, agrees with Eq. (87). On the other hand, for any temperature we have:

$$
\lim _{m \rightarrow 0}\langle\bar{\psi} \psi\rangle_{\beta}=0
$$

Thus, we see that the $(2+1)$-dimensional P-even massless fermion condensate in presence of a constant magnetic field is highly unstable. In fact the thermal condensate disappears as soon as a heat bath is introduced.

Let us, now, turn on the effective potential. Again we need to evaluate the Dirac energy $E_{D}$. To this end we rewrite the one-loop Hamiltonian Eq. (21) by using the expansion Eq. (84). For the Dirac Hamiltonian $H_{D}$ we find :

$$
\begin{aligned}
H_{D}= & \int d p E_{0}\left[a^{\dagger}(0, p) a(0, p)+b^{\dagger}(0, p) b(0, p)\right]+\sum_{n=1}^{\infty} \sum_{i=1,2} \int d p E_{n}\left[a_{i}^{\dagger}(n, p) a_{i}(n, p)+b_{i}^{\dagger}(n, p) b_{i}(n, p)\right] \\
& -\frac{e B}{4 \pi} V\left[E_{0}+2 \sum_{n=1}^{\infty} E_{n}\right] .
\end{aligned}
$$

As a consequence the Dirac energy defined by Eq. (40) is:

$$
E_{D}(B)=-\frac{e B}{2 \pi} \frac{V}{2}\left[E_{0}+2 \sum_{n=1}^{\infty} E_{n}\right]
$$

Proceeding as in the previous Section we define the vacuum energy density. We get:

$$
\tilde{\mathcal{E}}(B)=\mathcal{E}(B)-\mathcal{E}(0)=\frac{B^{2}}{2}+\frac{(e B)^{\frac{3}{2}}}{2 \pi} g\left(\frac{e B}{m^{2}}\right)+\frac{e B}{4 \pi}|m|,
$$


where the function $g(\lambda)$ is given by Eq. (47).

Note that, if we rewrite the last term on the right hand size of Eq. (93) by using the integral representation Eq. (47), and after some manipulations, we get:

$$
\tilde{\mathcal{E}}(B)=\frac{B^{2}}{2}+\frac{1}{8 \pi^{\frac{3}{2}}} \int_{0}^{\infty} \frac{d s}{s^{\frac{5}{2}}} e^{-m^{2} s}[(e B s) \operatorname{coth}(e B s)-1] .
$$

Equation (94) agrees with the calculation by Redlich [20].

As concern the finite temperature effective potential we follow the same steps as in the previous Section. The partition function is :

$$
Z_{0}=\operatorname{Tr} e^{-\beta\left(H_{D}+\frac{V B^{2}}{2}\right)}=e^{-\beta \frac{B^{2}}{2} V}\left(1-e^{-\beta E_{0}}\right)^{d} e^{\beta \frac{d}{4} E_{0}} \prod_{n=1}^{\infty}\left(1-e^{-\beta E_{n}}\right)^{4 d} e^{\beta \frac{d}{2} E_{n}}
$$

where $d=\frac{e B}{\pi}$. So that the free energy density is:

$$
\mathcal{F}_{0}(B)=-\frac{4 d}{\beta} \sum_{n=1}^{\infty} \ln \left(1+e^{-\beta E_{n}}\right)-\frac{d}{\beta} \ln \left(1+e^{-\beta E_{0}}\right)-\frac{d}{4} E_{0}-\frac{d}{2} \sum_{n=1}^{\infty} E_{n}+\frac{B^{2}}{2} .
$$

Subtracting the contribution at $B=0$, we obtain

$$
\begin{aligned}
\tilde{\mathcal{F}}_{0}(B) & =\mathcal{F}_{0}(B)-\mathcal{F}_{0}(B=0)= \\
& =4 \tilde{I}_{1}(B)-\frac{e B}{\pi \beta} \ln \left(1+e^{-\beta m}\right)+\frac{(e B)^{\frac{3}{2}}}{2 \pi} g\left(\frac{e B}{m^{2}}\right)+\frac{e B}{4 \pi}|m|+\frac{B^{2}}{2}
\end{aligned}
$$

where $\tilde{I}_{1}(B)$ has been already defined in the previous Section. Note that, as expected, the zero-temperature limit of Eq. (97) reduces to Eq. (93).

In Figure 7 we plot the free energy density as a function of $\lambda$ for three values of temperature $\hat{T}$. As it is evident the spontaneous generation of an uniform magnetic condensate is not energetically favorable neither at zero temperature nor at any finite temperature.

\section{Dynamical Generation of Mass in External Magnetic Field}

In this Section we investigate the spontaneous generation of the fermion mass in presence of an external magnetic field. Let us consider massless Dirac fermions in an external constant magnetic field. The relevant Lagrangian is now : 


$$
\mathcal{L}_{Q E D}(x)=\bar{\psi}(x) \gamma^{\mu}\left(i \partial_{\mu}-e A_{\mu}\right) \psi(x)-\frac{1}{4} F_{\mu \nu}(x) F^{\mu \nu}(x)
$$

Recently there has been renewed interest in the dynamical symmetry breaking in $(2+1)$ dimensional $Q E D$. In particular it turns out that in planar $Q E D$ a constant magnetic field leads to the dynamical generation of a P-even mass for four-component Dirac fermions [21]. In order to investigate the dynamical symmetry breaking we must consider the effective action for composite operators [22]. For the problem of spontaneous generation of a fermion mass the effective action will depend on the complete fermion propagator of the theory. However we restrict ourselves to the case of a constant external source coupled to the local operator $\psi \overline{(x)} \psi(x)$.

In this case the external source acts like a fermion mass $m$, so that the effective action depends on the full propagator for massive fermion fields. Moreover, owing to the translation invariance of the problem, we need to consider the effective potential. According to Ref. [22], the relevant quantity turns out to be:

$$
\Omega(B, m)=V_{\mathrm{eff}}(B, m)-V_{\mathrm{eff}}(B, 0),
$$

where $V_{\text {eff }}(B, m)$ is the effective potential in presence of both the magnetic field and the constant fermion mass. Moreover we have the well known physical interpretation that:

$$
V_{\text {eff }}(B, m)=\frac{1}{V} \frac{\left\langle 0_{m}|H| 0_{m}\right\rangle}{\left\langle 0_{m} \mid 0_{m}\right\rangle}
$$

where $\left|0_{m}\right\rangle$ indicates the ground state of the massive theory, and $H$ is the massless Hamiltonian:

$$
H=\int d^{2} x\left[\frac{B^{2}}{2}+\psi^{\dagger}(-i \vec{\alpha} \cdot \vec{\nabla}-e \vec{\alpha} \cdot \vec{A}) \psi\right]
$$

Our strategy is to consider a trial vacuum state which depends on the variational parameter $m$, and thus determine the effective potential by minimizing the vacuum energy density. We are interested in the case of a given uniform external magnetic field. In this case, in the one-loop approximation the Hamiltonian reduces to: 


$$
H=\int d^{2} x\left[\frac{B^{2}}{2}+\psi^{\dagger}(-i \vec{\alpha} \cdot \vec{\nabla}-e \vec{\alpha} \cdot \overrightarrow{\vec{A}}) \psi\right]
$$

where $\overrightarrow{\bar{A}}$ is given by Eq. (201). To evaluate the effective potential we need to calculate the expectation value of the massless Hamiltonian Eq. (102) on the massive fermion ground state.

The case of P-even mass term has been extensively discussed in the literature [21]. Therefore we restrict to the case of P-odd Dirac fermions.

Let us consider, firstly, the case of a negative mass term $m=-|m|$. The massive ground state $\left|0_{m}\right\rangle$ have been already constructed in Sect. 2. Using the results of that Section we find:

$$
\frac{1}{V} \frac{\left\langle 0_{m}|H| 0_{m}\right\rangle}{\left\langle 0_{m} \mid 0_{m}\right\rangle}=\frac{B^{2}}{2}-\frac{e B}{2 \pi} \sum_{n=0}^{\infty} \sqrt{2 n e B+m^{2}}+\frac{|m|}{V} \frac{\left\langle 0_{m}|\bar{\psi}(\vec{x}) \psi(\vec{x})| 0_{m}\right\rangle}{\left\langle 0_{m} \mid 0_{m}\right\rangle}
$$

From Eq. (36) it follows:

$$
\frac{1}{V} \frac{\left\langle 0_{m}|H| 0_{m}\right\rangle}{\left\langle 0_{m} \mid 0_{m}\right\rangle}=\frac{B^{2}}{2}-\frac{e B}{2 \pi} \sum_{n=1} \sqrt{2 n e B+m^{2}}+m^{2} \frac{e B}{2 \pi} \sum_{n=1}^{\infty} \frac{1}{\sqrt{2 n e B+m^{2}}}
$$

It easy to check that the same result follows in the case of positive mass term. So that we get

$$
V_{\text {eff }}(B, m)=\frac{B^{2}}{2}-\frac{e B}{2 \pi} \sum_{n=1}^{\infty} \sqrt{2 n e B+m^{2}}+\frac{m^{2} e B}{2 \pi} \sum_{n=1}^{\infty} \frac{1}{\sqrt{2 n e B+m^{2}}} .
$$

Observing that

$$
V_{\text {eff }}(B, 0)=\frac{B^{2}}{2}+\frac{\sqrt{2}}{8 \pi^{2}} \zeta\left(\frac{3}{2}\right)(e B)^{\frac{3}{2}}
$$

we get finally

$$
\Omega(B, m)=-\frac{e B}{2 \pi} \sum_{n=1}^{\infty} \sqrt{2 n e B+m^{2}}+\frac{m^{2} e B}{2 \pi} \sum_{n=1}^{\infty} \frac{1}{\sqrt{2 n e B+m^{2}}}-\frac{\sqrt{2} \zeta\left(\frac{3}{2}\right)}{8 \pi^{2}}(e B)^{\frac{3}{2}} .
$$

Note that, as expected, the effective potential $\Omega(B, m)$ depends on $|m|$. By using the integral representation Eq. (43) together with

$$
\frac{1}{\sqrt{a}}=\int_{0}^{\infty} \frac{d s}{\sqrt{\pi s}} e^{-a s}
$$


we obtain the following integral representation for the effective potential:

$$
\Omega(B, m)=|m|^{3}\left[J(\lambda)-\frac{\sqrt{2}}{8 \pi^{2}} \zeta\left(\frac{3}{2}\right) \lambda^{\frac{3}{2}}\right]
$$

where we recall that $\lambda=\frac{e B}{m^{2}}$ and

$$
J(\lambda)=-\frac{\lambda^{2}}{\pi} \int_{0}^{\infty} \frac{d y}{\sqrt{\pi y}} e^{y}\left[\frac{e^{-2 \lambda y}}{\left(1-e^{-2 \lambda y}\right)^{2}}-\frac{1}{(2 \lambda y)^{2}}\right]
$$

In order to obtain the small $|m|$ behaviour of the effective potential, we note that

$$
J(\lambda) \stackrel{\lambda \rightarrow \infty}{\sim} \frac{\sqrt{2}}{8 \pi^{2}} \lambda^{\frac{3}{2}} \zeta\left(\frac{3}{2}\right)+\frac{\sqrt{2}}{8 \pi} \lambda \zeta\left(\frac{1}{2}\right)+\mathcal{O}\left(\frac{1}{\sqrt{\lambda}}\right) .
$$

So that for small $|m|$ we get

$$
\Omega(B, m)=\frac{\sqrt{2}}{8 \pi} \zeta\left(\frac{1}{2}\right) \sqrt{e B} m^{2}+\mathcal{O}\left(m^{4}\right)
$$

On the other hand for large $|m|$ we find

$$
J(\lambda) \stackrel{\lambda \rightarrow 0}{\sim} \frac{\lambda^{2}}{12 \pi}+\frac{\lambda^{3}}{6 \pi}+\mathcal{O}\left(\lambda^{4}\right)
$$

so that

$$
\Omega(B, m)=-\frac{\sqrt{2}}{8 \pi^{2}} \zeta\left(\frac{3}{2}\right)(e B)^{\frac{3}{2}}+\mathcal{O}\left(\frac{1}{|m|}\right)
$$

Equation (112) tell us that the one-loop effective potential $\Omega(B, m)$ displays an absolute maximum at $m=0$. Moreover it turns out that $\Omega(B, m)<0$ (see Fig. 8). So that it is energetically fovourable to generate a constant fermion mass.

Remarkably, a straightforward calculation shows that in the one-loop approximation the effective potential $\Omega(B, m)$ given by Eq. (107) accounts for the dynamical generation of a constant mass also in the case of four component fermions. As a consequence the remarkable phenomenon of the dynamical generation of a P-even fermion mass in presence of an external constant magnetic fields displays itself even in the one-loop approximation. Note that the minimum of the one-loop effective potential is obtained for infinite value of the mass. However, we feel that this result is an artefact of the one-loop approximation. 
In fact, including the higher-order corrections should give rise to a negative minimum for finite value of the fermion mass. It is worthwhile to briefly compare our approach with the papers in Ref [21]. The papers in Ref [21] deal with four-component P-even Dirac fermions coupled with $(2+1)$-dimensional electromagnetic fields. In these papers it is studied the full Schwinger-Dyson equations in the limit of strong magnetic field where the lowest Landau level accounts for the fermion dynamics. On the other hand, in our approach we restrict to the one-loop approximation, but we take into account all Landau levels, reaching conclusion similar to the ones in Ref [21]. Thus we feel that our study corroborates the validity of the approximation of Ref [21].

\section{Conclusions}

In this paper we have discussed in details the dynamics of $(2+1)$-dimensional Dirac fermions coupled to an external magnetic field in the one-loop approximation both at zero and finite temperature.

In particular we investigated in the two formulations the spontaneous generation af the magnetic condensate both at $T=0$ and $T \neq 0$. Our results show that in the one-loop approximation the remarkable phenomenon of spontaneous generation of an uniform magnetic condensate takes place only in the case of Dirac fermions with negative P-odd mass. Moreover the magnetic condensation survives the thermal corrections even at infinite tem-

perature. In addition at high temperature the thermal fluctuations tend to increase the condensation energy and the strength of the induced magnetic field .

It is worthwhile to note that the effective system composed of half filled zero modes at zero temperature accounts for the high temperature limit of the free energy. So that we see that the dynamics of the zero modes is responsible for the dynamical magnetic condensation both at zero and high temperatures. Remarkably, as discussed in the Introduction, it turns out that P-odd Dirac fermions are relevant for the descriptions of zero modes localized on a domain wall in the case of four dimensional fermions coupled with a scalar field. Indeed it 
has been suggested [9] that the spontaneous generation of an uniform magnetic condensate gives rise to ferromagnetic domains walls at the electroweak phase transition . This, in turns, may have far reaching consequences during the electroweak phase transition in the early universe.

We also showed that even in the one-loop approximation a constant magnetic field is a strong catalyst for the spontaneous generation of a fermion mass. In particular we find that the spontaneous generation of a constant fermion mass does not depend on the spinorial representation for the Dirac matrices. So that an external constant magnetic field induces the generation of both a constant P-even and P-odd fermion mass.

Finally, we discussed the massless limit of the fermion condensate for both P-even and Podd Dirac fermions in an external magnetic field. For both formulations we find that the massless limit of the condensate is non-analytic. Remarkably, it turns out that in presence of a heat bath, even with vanishing temperature, the fermion condensate survives to the massless limit only for P-odd two-component fermion fields. Moreover, in that case the massless limit is symmetric.

In conclusions, our results show that, within the one-loop approximation, the unique theory displaying a non-trivial ground state turns out to be the three-dimensional quantum electrodynamics in interaction with two-component Dirac fermion fields. 


\section{REFERENCES}

[1] R.Jackiw and S. Templeton, Phys. Rev. D 23 (1981) 2291; J. Schonfeld, Nucl. Phys. B 185 (1981) 157; S. Deser, R. Jackiw and S. Templeton, Ann. Phys. 140 (1982) 372.

[2] See for instance: E. Fradkin, Field Theories of Condensed Matter Systems, Addison Wesley, 1991.

[3] For a review, see: R. Jackiw, Massive gauge theories in three-dimensions (= at high temperature) presented at Artic School of Phys., Akaslompolo, Finland, 1982.

[4] I.J.R. Aitchison and N. Mavromatos, Phys. Rev. B 53 (1996) 9321.

[5] P. Cea, Phys. Rev. D 32 (1985) 2785; Phys. Rev D 34 (1986) 3229.

[6] Y. Hosotani, Phys. Lett. B 319 (1993) 332; Phys Rev. D 51 (1995) 2022.

[7] P. Cea, Phys. Rev. D 55 (1997) 7985.

[8] R. Jackiw and C.Rebbi, Phys. Rev D 13 (1976) 3398; A. T. Niemi and G.W. Semenoff, Phys. Rep. C 135 (1986) 100 .

[9] P. Cea and L. Tedesco Phys. Lett. B 450 (1999) 61.

[10] S. Kanemura and T. Matsushita, Phys. Rev. D 56 (1997) 2435.

[11] P. Cea and L. Tedesco Phys. Lett. B 425 (1998) 345.

[12] T.W. Appelquist, M. Bowick, D. Karabali and L. C.R. Wijewardhana Phys. Rev. D 33 (1986) 3704 .

[13] See, for instance : A.I. Akheizer and V.B. Berestetsky, Quantum Electrodynamics (Interscience, N,Y. 1965).

[14] A. Das and M. Hott, Phys. Rev. D 53 (1996) 2252.

[15] A. Niemi and G. Semenoff, Nucl. Phys. B 251 (1995) 15. 
[16] I.S. Gradshteyn and I.M. Ryzshik, Table of Integrals, Series and Products.

[17] T. Appelquist, M. Bowick, D. Karabali, and L.C.R. Wijewardhana, Phys. Rev. D 33 (1986) 3704 .

[18] J. Schwinger, Phys. Rev. 82 (1951) 664.

[19] H. Umezawa, H. Matsumoto and H. Tachiki, Thermo Field Dynamics, Noth-Holland, Amsterdam, 1982.

[20] A.N. Redlich, Phys. Rev. D 29 (1984) 2366.

[21] V. Gusynin, V. Miransky and I. Shovkovy, Phys. Rev. D 52 (1996) 4718; Nucl. Phys. B 462 (1995) 249; A.V. Shpagin, Dynamical mass generation in $(2+1)$-dimensional electrodynamics in an external magnetic field, hep-ph/9611412; T. Itoh and H. Kato, Dynamical Generation of fermion Mass and Magnetic Field in Three-Dimensional QED with Chern-Simon Term, hep-th/9802101; K. Farakos, G. Koutsoumbas, N.E. Mavromatos and A. Momen, Catalysis of Chiral Symmetry Breaking by External Magnetic Fields in Three Dimensional Lattice QED hep-lat/9902017; K. Farakos and Mavromatos, Int. J. Mod. Phys. B 12 (1998) 809; K. Faros, G. Koutsoumbas and N.E. Mavromatos, Phys. Lett. B 431 (1998) 147.

[22] J.M. Corwall, R. Jackiw and E. Tomboulis, Phys. Rev. D 10 (1974) 2428. 

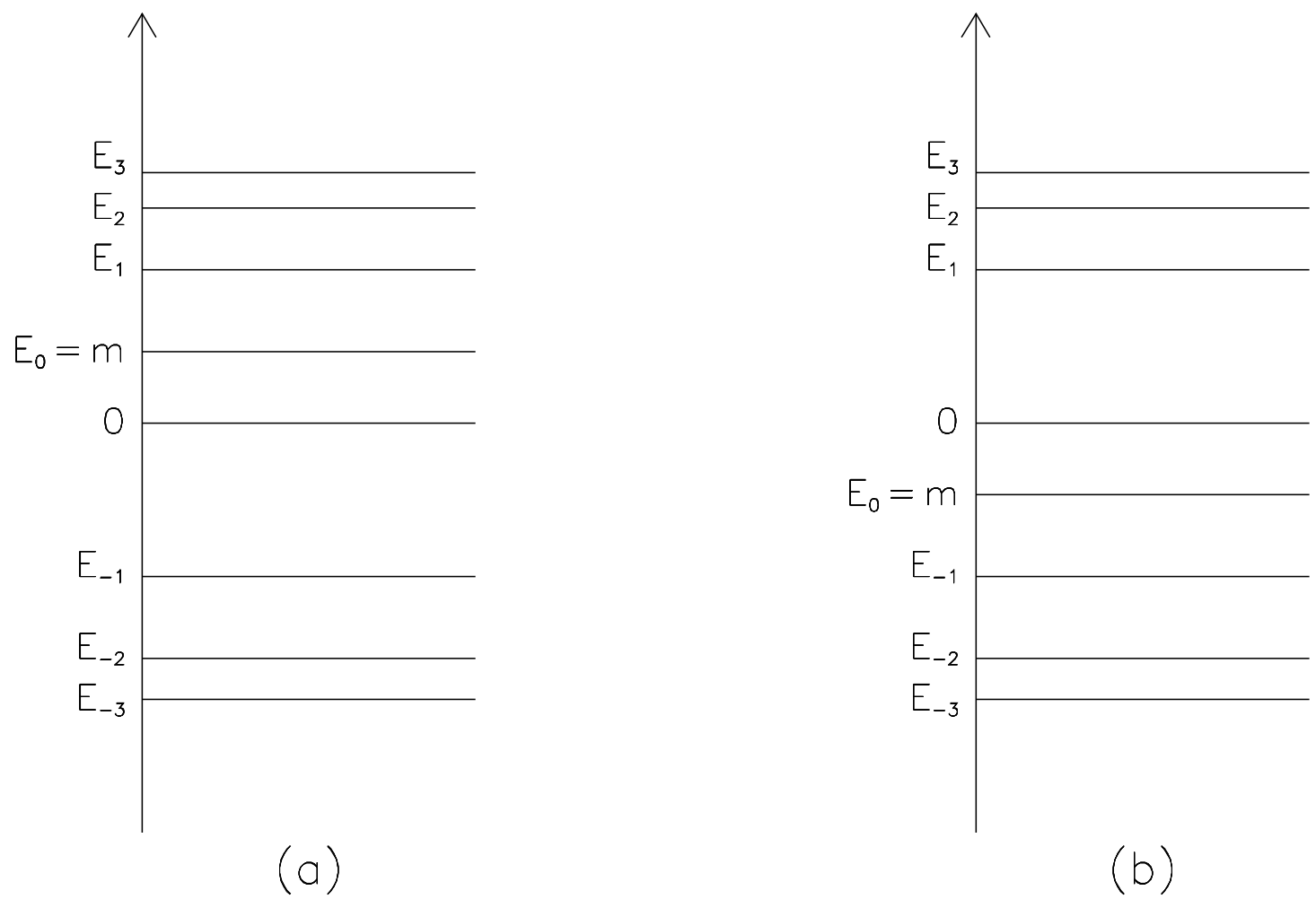

(b)

FIG. 1 The Landau levels for Dirac fermions in a constant magnetic field: $a) m>0$ and b) $m<0$. 


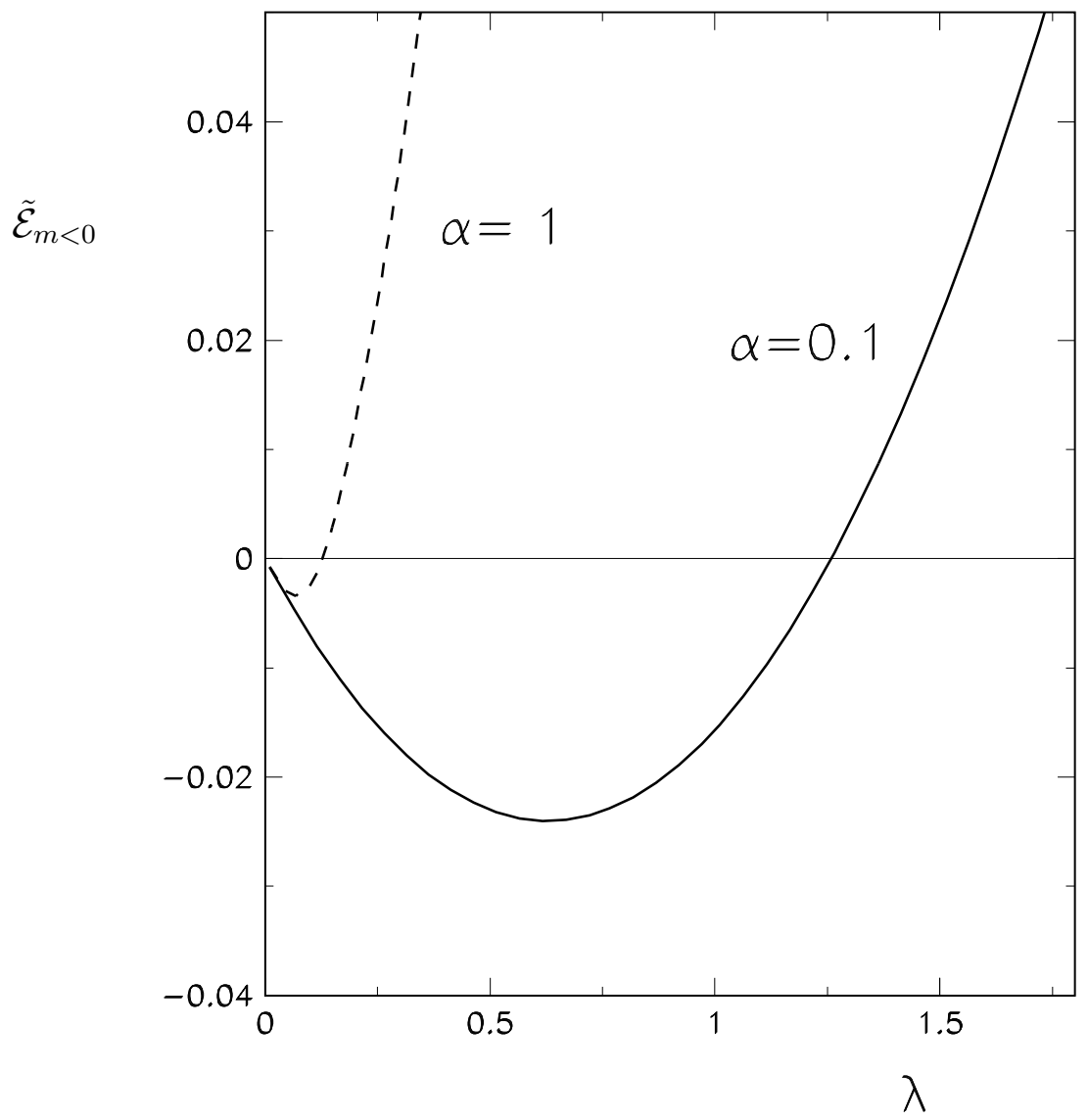

FIG. 2 Plot of the energy density $\frac{\tilde{\mathcal{E}}_{m<0}}{|m|^{3}}$ as a function of $\lambda$ for two values of $\alpha=\frac{|m|}{e^{2}}$ : full line $\alpha=0.1$, dashed line $\alpha=1$. 


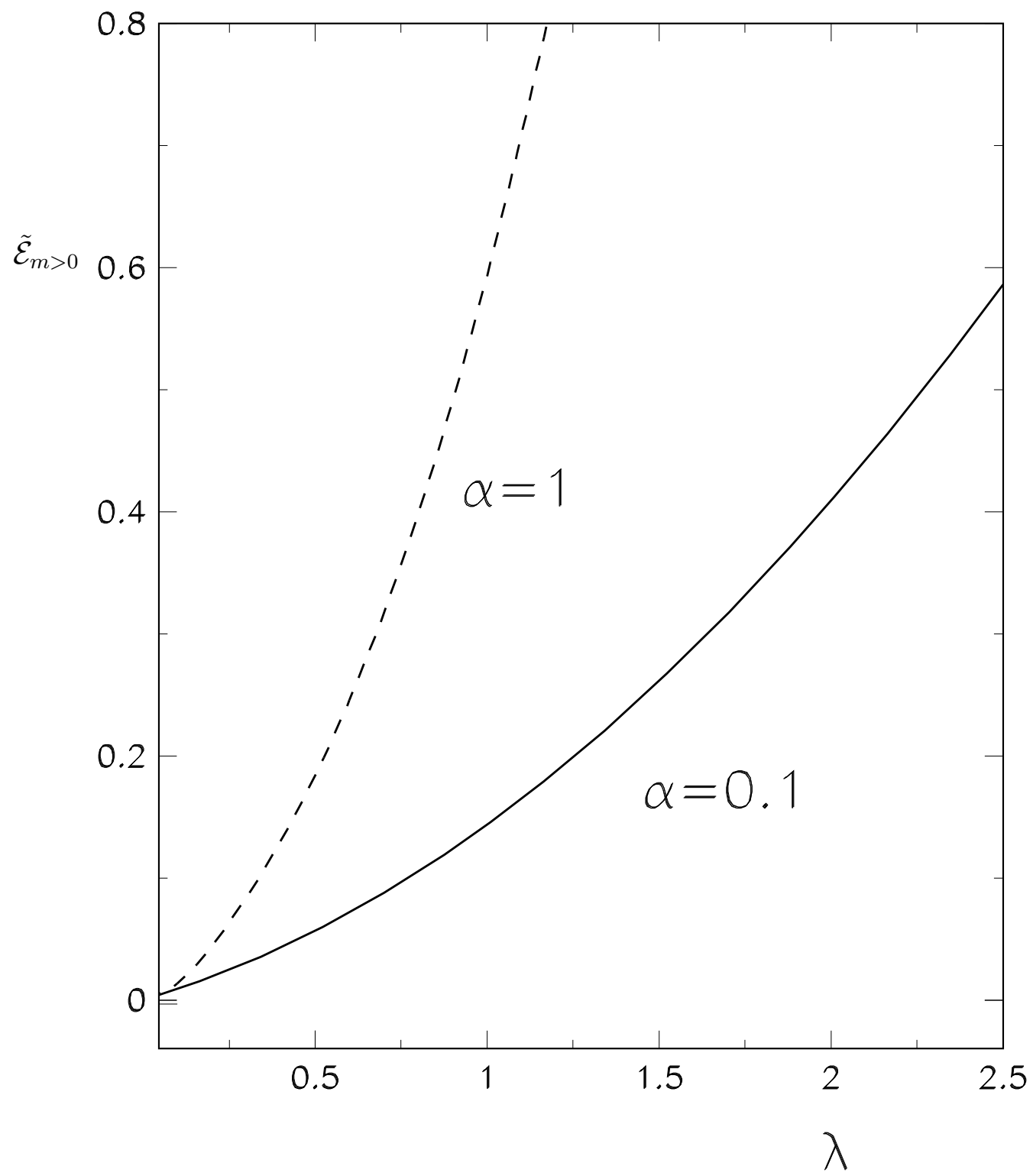

FIG. 3 Plot of the energy density $\frac{\tilde{\mathcal{E}}_{m>0}}{|m|^{3}}$ as a function of $\lambda$ for two values of $\alpha=\frac{|m|}{e^{2}}$ : full line $\alpha=0.1$, dashed line $\alpha=1$. 


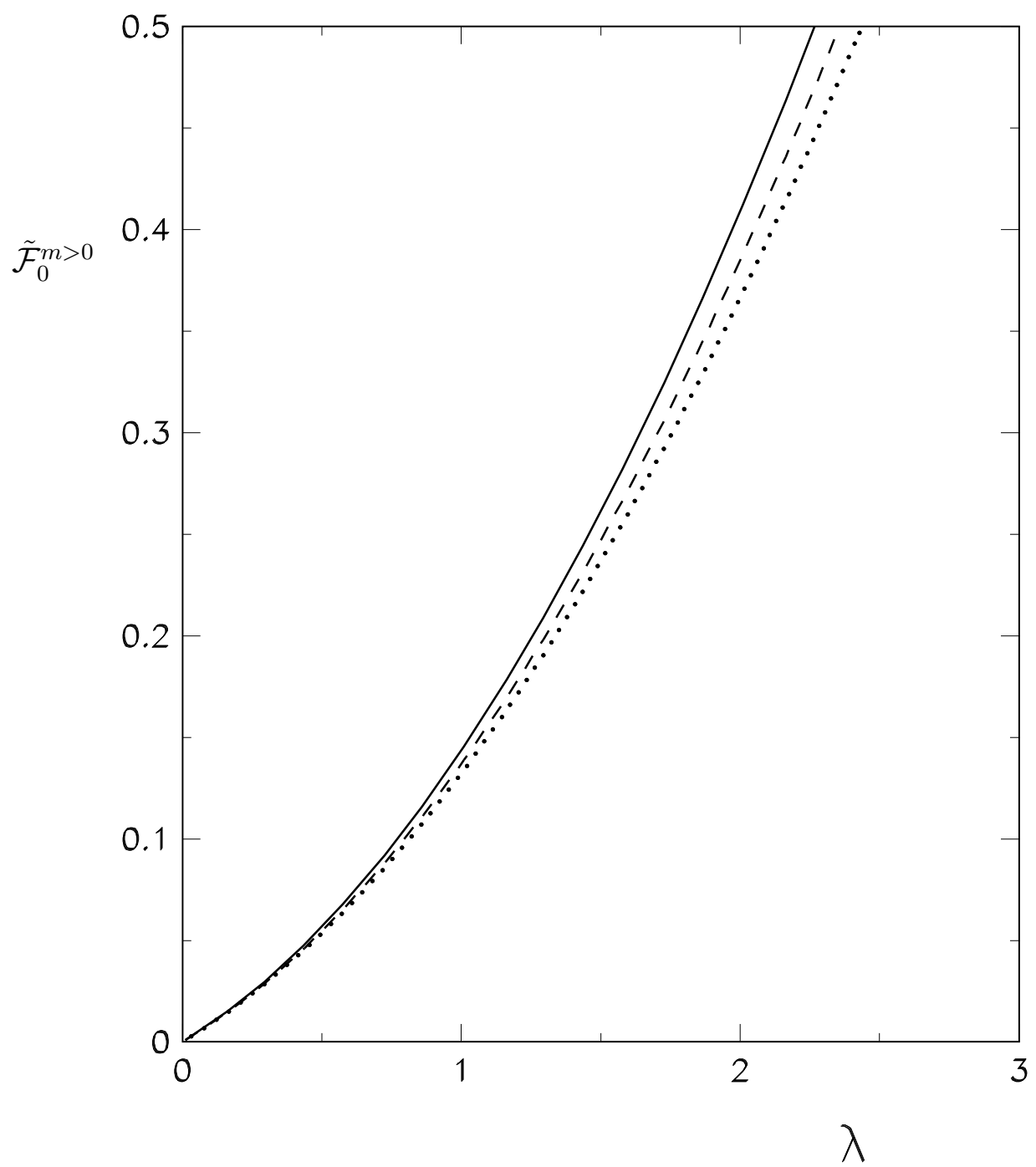

FIG. 4 Free energy density (in units of $|m|^{3}$ ) versus $\lambda$ for $\alpha=0.1$ and $m>0$. Full line $T=0$, dashed line $\hat{T}=1$, dotted line $\hat{T}=5$. 


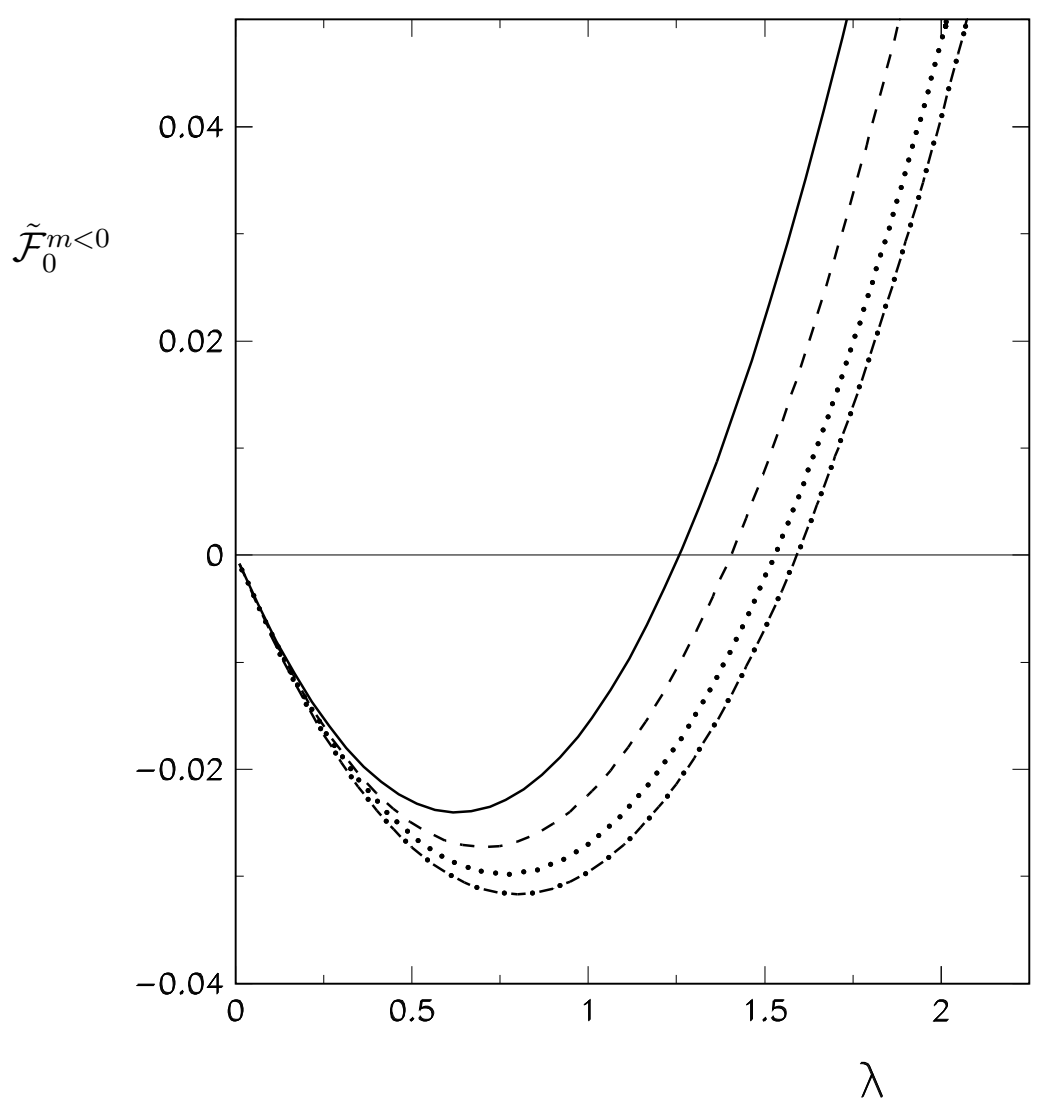

FIG. 5 Free energy density (in units of $|m|^{3}$ ) versus $\lambda$ for $\alpha=0.1$ and $m<0$. Full line $T=0$, dashed line $\hat{T}=1$, dotted line $\hat{T}=5$ and dashed-dotted line $\hat{T} \rightarrow \infty$. 


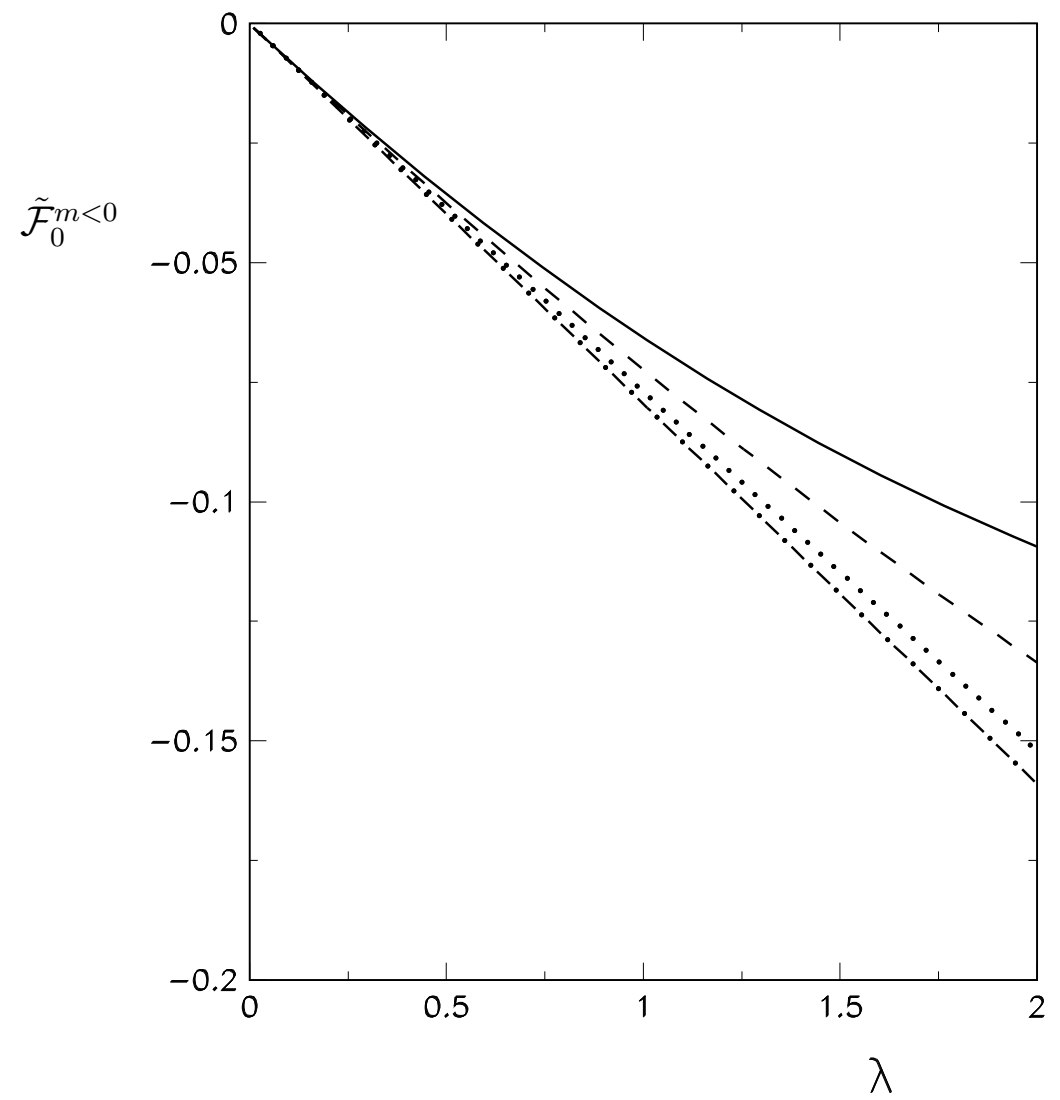

FIG. 6 Free energy density (in unit of $|m|^{3}$ ) versus $\lambda$ for $\alpha=0$ and $m<0$. Temperature values as in FIG. 5. 


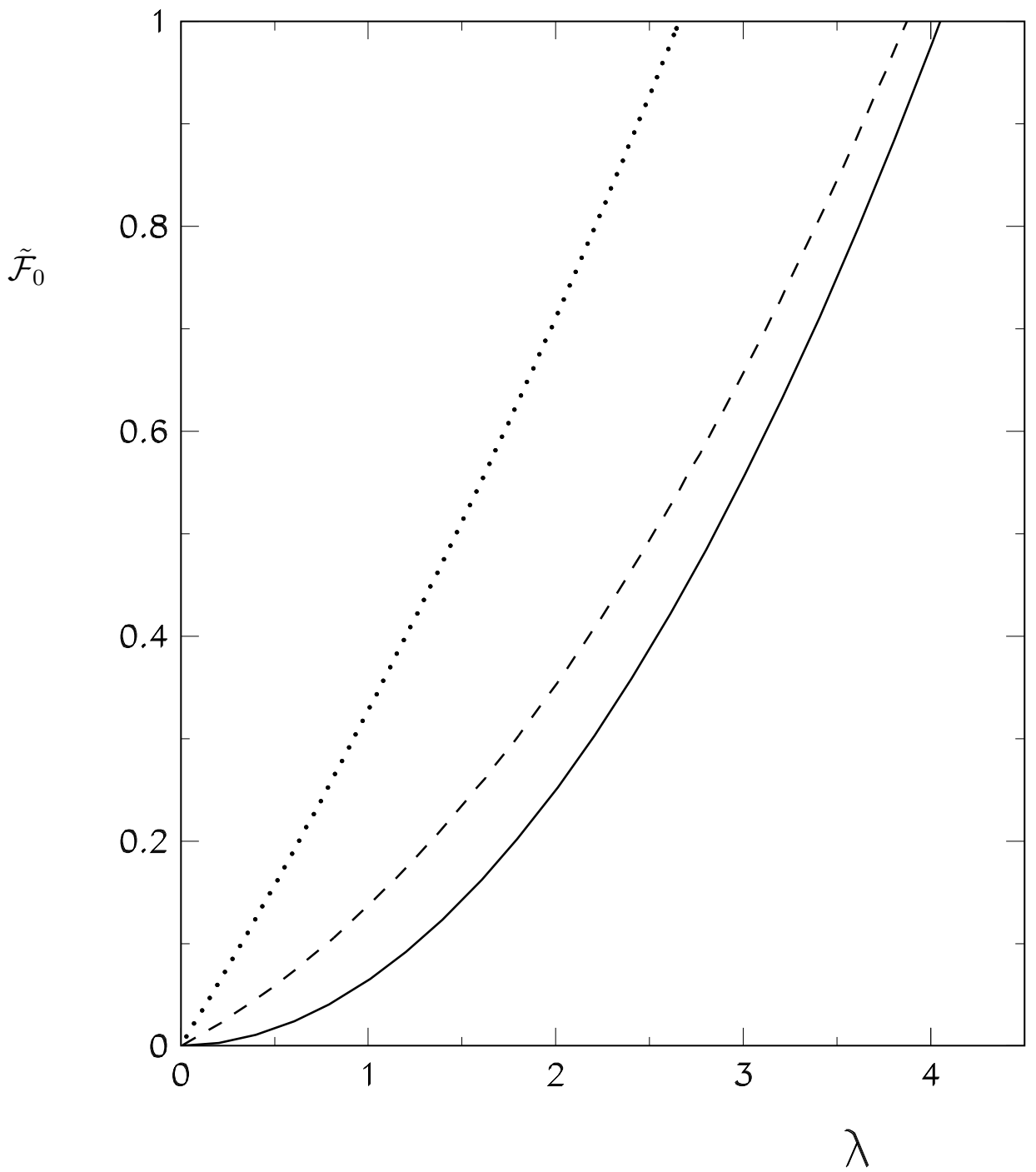

FIG. 7 Free energy density in the P-even formulation (in units of $|m|^{3}$ ) versus $\lambda$ for $\alpha=0.1$. Full line $T=0$, dashed line $\hat{T}=1$, dotted line $\hat{T}=2$. 


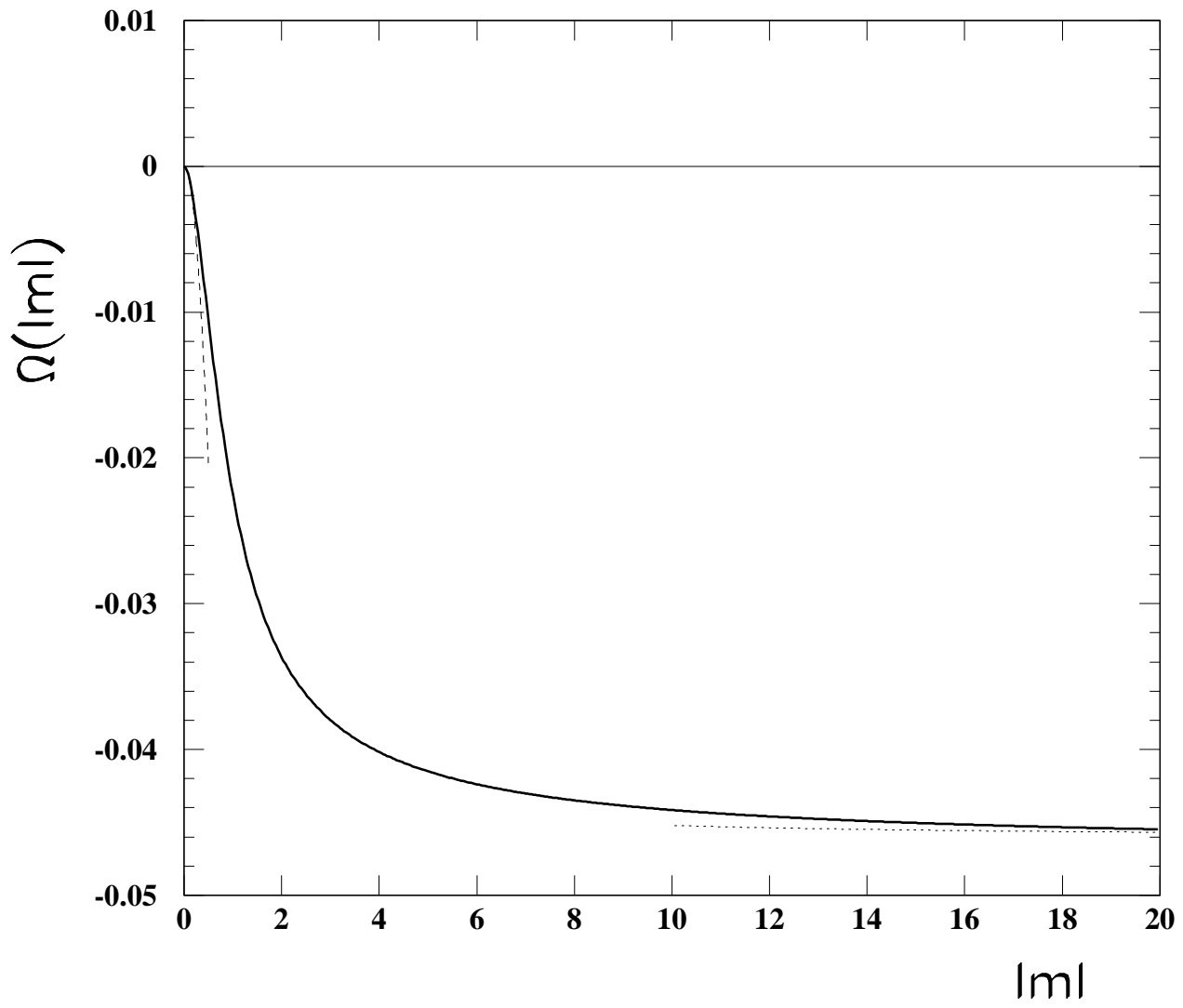

FIG. 8 The effective potential $\Omega(B, m)$ versus $|m|$ for $e B=1$. Dashed and dotted lines are the small and large $|m|$ expansions respectively. 